\title{
Non-rigid Registration Using Morphons
}

\author{
Andreas Wrangsjö, Johanna Pettersson, and Hans Knutsson \\ Medical Informatics, Department of Biomedical Engineering, \\ and Center for Medical Image Science and Visualization, \\ Linköping University, Sweden
}

\begin{abstract}
The Morphon, a non-rigid registration method is presented and applied to a number of registration applications. The algorithm takes a prototype image (or volume) and morphs it into a target image using an iterative, multi-resolution technique. The deformation process is done in three steps: displacement estimation, deformation field accumulation and deformation. The framework could be described in very general terms, but in this paper we focus on a specific implementation of the Morphon framework. The method can be employed in a wide range of registration tasks, which is shown in four very different registration examples; $2 \mathrm{D}$ photographs of hands and faces, 3D CT data of the hip region, and 3D MR brain images.
\end{abstract}

\section{Introduction}

Image registration is a necessary process in many applications, for example when fusing images from different medical imaging modalities, or correcting geometrically distorted or disaligned images. The goal with the registration procedure is to find a transformation from one image (or volume) to another, such that the difference between the the deformed image and the target image is minimised in some sense. There exist a considerable amount of methods for solving this problem. It is not straightforward how to classify different registration methods. They differ in the types of image features they work on, the way they measure similarity, the optimisation scheme, and the types of deformations they allow. A good survey can be found in e.g. [9].

There are several ways to choose what image features to compare. The most basic procedure is to simply compare the image intensity between the two images. More elaborate features are based on intensity gradients, local structure tensors or geometrical landmarks. Furthermore, a way to find the correspondence between the image features must be introduced. This measure should be chosen carefully and with the application in mind to give a good measure of the similarity between the images. Common methods are based on (normalised) cross correlation or mutual information between the image features [8]. With a similarity measure the registration process can be stated as an optimisation problem, or, on the other hand, as a minimsiation of the dissimilarity. The transformation model defines how the registered image is allowed to deform. These are typically referred to as global/local or rigid/non-rigid models. A global model 
implies that the same transformation model is applied to the entire image, while a local model indicates that different parts of the image may have different transformation models. The rigidity of the model refers to the degrees of freedom of the transformations. A rigid model has a very low number of degrees of freedom and a non-rigid model allows for a higher amount of deformation.

\section{The Morphon Method}

The Morphon essentially attempts to iteratively deform a prototype image (or volume) into a target image by morphing the prototype. The process can be divided into three steps: displacement estimation, deformation field accumulation and deformation.

- The displacement estimation aims to find local indications on how to deform the prototype to make it more similar to the target image. In the implementation presented here, these estimates are based on quadrature phase differences.

- The deformation field accumulation uses the displacement estimates to update a deformation field. This step involves two processes: updating the accumulated deformation field and regularisation of the displacement estimates and/or accumulated field. This regularisation is used to fit the observed deformation to a local and/or global deformation model.

- Finally, the deformation morphs the original prototype according to the accumulated deformation field. In this implementation conventional bi/trilinear interpolation has been used for this purpose.

These three steps are iterated for as long as the displacement estimates indicate further morphing to be beneficial. Furthermore, a scale space scheme has been included in the Morphon. This enables successful morphing also between quite dissimilar images.

The method is so far indeed quite general. Its usability is, however, quite dependent on what methods one makes use of in following the steps above. The remainder of this section is devoted to the methods we have chosen in our implementation.

\section{Quadrature Phase Difference}

Estimating optical flow, motion, orientation or displacement between images are essentially equivalent problems. Being very typical image analysis issues, a multitude of methods have evolved to cope with them. There are, among others, methods based on gradient [7,1], polynomial expansions [2] and quadrature phase $[4,5,6])$. We have chosen to use quadrature phase differences to estimate the local displacement between the two images. Among the benefits of this method are invariance to image intensity and weak gradients.

Quadrature phase can be described as a measure of local structure. Edges between dark and bright areas in the image have one phase, lines on dark background have one, bright patches have one and dark lines have one. The transition 
between local phase values is continuous as we move e.g. from a dark patch across an edge and into a bright patch. The difference in local phase between prototype and target images is therefore a good measure of how much the prototype needs to be moved (locally) to fit the target image. The phase is, however, by definition one-dimensional. For multi-dimensional signals this means that the phase needs to either be associated with a certain direction [5] or redefined to be meaningful in a multi-dimensional sense [3]. By using a set of multidimensional quadrature filters, each associated with a direction and an angular function, we can obtain phase and magnitude estimates corresponding to the filter directions. If these are chosen carefully and the angular functions are chosen to constitute a spherical harmonics basis, we can estimate any local orientation and phase of the image. If each region of the image was a simple neighbourhood, where the image intensity could be described as a function of only one variable, we would be able to find a perfect estimate of the local orientation and an ideal measure of local phase (given the spatial frequency function of the filters). The local displacement is then found as a function of the local phase along its associated direction. Images are not simple, however, and we need to settle with an approximation of the local phase and its direction. Here, we have used a least square estimate of the local displacement.

$$
\min _{\mathbf{v}} \sum_{i}\left[w_{i}\left(\hat{\mathbf{n}}_{i}^{T} \mathbf{v}-v_{i}\right)\right]^{2}
$$

where $\mathbf{v}$ is the sought displacement field estimate, $\hat{\mathbf{n}}_{i}$ is the direction of filter $i$, $v_{i}$ is the displacement field associated to filter direction $i$, and $w_{i}$ is a certainty measure, derived from the magnitude of the phase difference. By using this estimation scheme we obtain a fairly good estimate of local displacement. Note, however, that these estimates are always perpendicular to the local structure. This is due to the aperture problem. If the images are e.g two lines of the same orientation, we can only estimate the orthogonal distance between the lines. Displacement along the lines is, and will always be, impossible to estimate.

Using quadrature phase in displacement estimation gives us a quite simple and useful certainty measure, briefly mentioned in Eq. (1) above. If the filter response magnitude at some position in the image is large, we are more inclined to trust that phase estimate than if it is small. It is thus beneficial a function of the magnitude as certainty for the displacement estimates.

\section{Deformation}

Eq. (1) gives us a displacement field for the current iteration and scale. The displacement field should be used to interpolate a new, deformed version of the prototype. However, the interpolation step introduces small errors in the data. To avoid these errors to escalate when the prototype is iteratively interpolated, we accumulate the displacement fields into one total field that represents how the original prototype should be morphed. For each iteration the original prototype is deformed according to the accumulated field and then compared to the target data to estimate a displacement field for the current iteration. 
The updated accumulated field, $\mathbf{d}_{a}^{\prime}$, is obtained by combining the accumulated field, $\mathbf{d}_{a}$, and the displacement field from the current iteration, $\mathbf{d}_{k}$. This accumulation also includes certainty measures for both the accumulated field, $c_{a}$, and the temporary field, $c_{k}$.

$$
\mathbf{d}_{a}^{\prime}=\frac{\mathbf{d}_{a} c_{a}+\left(\mathbf{d}_{a}+\mathbf{d}_{k}\right) c_{k}}{c_{a}+c_{k}}
$$

\section{Regularisation}

The local displacement estimates could be interpreted as local forces trying to pull the prototype their way. If we would simply deform the prototype by moving each pixel as suggested by the displacement estimate, we would surely end up with a completely disrupted image. Hence, there is need for regularisation of the displacement estimates.

Our deformation field can be regularised in a multitude of ways. As described in the introducing section, registration methods incorporate a great variety of deformation models. We could, e.g. find a (weighted) least squares fit to some global deformation model. In the simplest case, we could fit it to a global rotation and translation model. This would correspond to rigid registration. The only allowed deformations would be those that can be described as a global rotation and/or translation. We could also give the model more degrees of freedom. Scaling, skewing, etc. could be added to our global model. The model could also be regional, e.g. a FEM model, where the observed displacements would be fitted according to their nearest landmark (node) points in a mesh model. The model could also be local. That is, we can fit the displacement estimates locally according to some neighbourhood function. The simplest case is to perform a local averaging of the estimates. The regularisation method could also be altered or modified during the registration. We could e.g. start out using a global model to find a good initial deformation. The regularisation could then be adapted towards a local model after a few iterations. Thus the benefits of global and local registration can be incorporated in the same model.

\section{Certainty Measures}

As mentioned earlier, certainty measures can be computed for the displacement estimates. Typically, edges, lines and surfaces are structures where the quadrature filters give strong responses. Using the response amplitude as a certainty measure would, thus, yields large certainties in such regions. If e.g. a global least squares fit to some deformation model is used in the regularisation, these certainties are used as weights in weighted LSQ. If, on the other hand, we use a local averaging or similar, the conventional averaging convolution operator is replaced by normalised averaging [5].

\section{Scale Spaces}

Local displacement is usually scale dependent. It is perfectly possible for two images to be horizontally displaced when observed on a fine scale but vertically 
if you look on a coarse scale. A typical problem in registration is to know what features of the prototype image to associate with what features of the target image. Our approach is to start comparing the images on a very coarse scale. Once the images are registered on that scale we move on to a finer scale. This simple scale space scheme has proven to be quite successful.

\section{$3 \quad$ Experiments}

To demonstrate some of the capabilities of Morphon registration, a set of four quite different registration tasks are presented along with experimental results using the Morphon.

\section{Hands}

A set of hands were photographed. Since the hands belong to different people, there are differences in size and shape. There are also differences in pose between the photos. Clearly, any rigid registration would fail in this case. Two experiments were carried out on the hands. The first one makes use of a local normalised Gaussian averaging model in the regularisation of the deformation field. The second uses a global affine regularisation model (which limits the allowed deformation quite a lot more than the Gaussian one). Figures 1 and 2 show the results when using the normalised Gaussian averaging and figures 3 and 4 show the same results using the affine model.

\section{Faces}

The next example deals with registration of images of faces. We show results both for registration between the faces of two different persons, Fig. 5, as well as registration of the same face but with different facial expression, Fig. 6. In both cases, a local transformation model based on local normalised Gaussian averaging, has been used. This example is more difficult than the hand example. Here, the images consist of objects that do not necessarily look at all the same. Even in the case where the same person is used as both target data and prototype data the images can look quite different just by changing the pose somewhat. An ear might show in one image and not in the other.
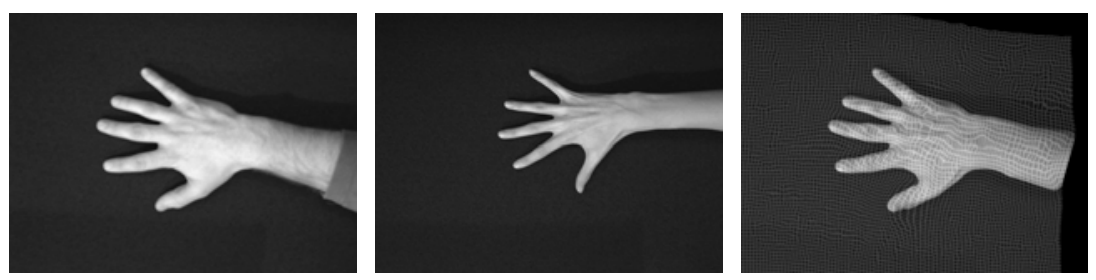

Fig. 1. Left: The target data hand, Middle: The hand to be deformed, Right: The deformed hand (with an added grid to show the deformation). Here, a normalised Gaussian regularisation has been used 


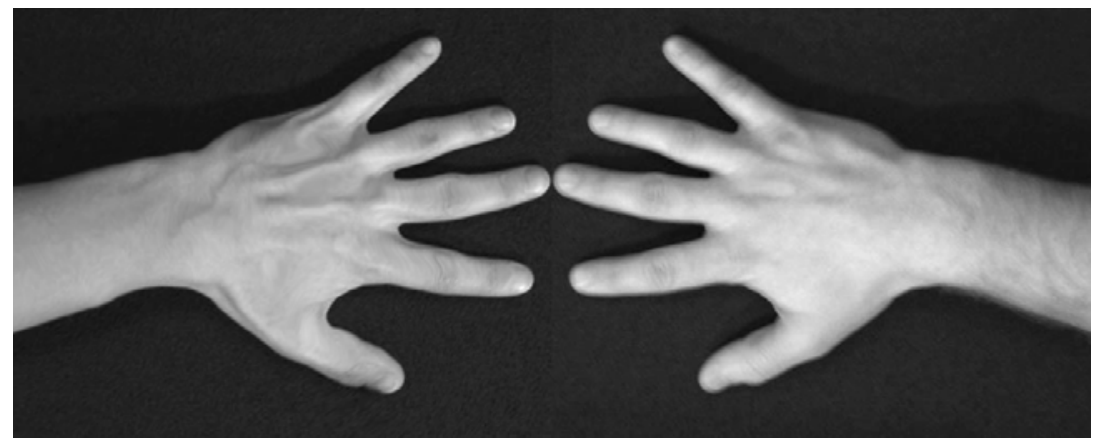

Fig. 2. The results from normalised Gaussian hand registration. Left: The registered hand (mirrored), Right: The 'real' hand
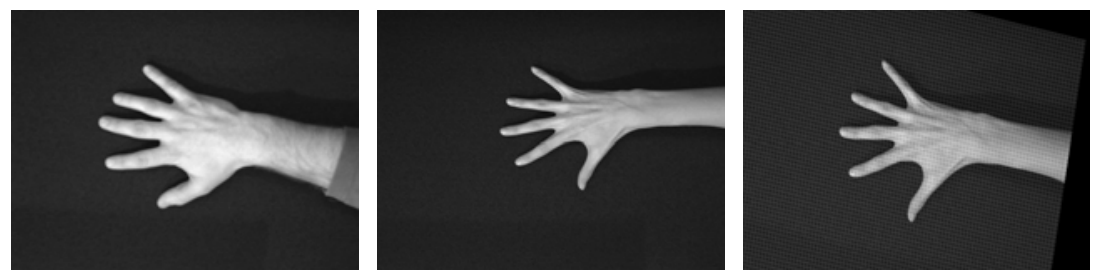

Fig. 3. Left: The target data hand, Middle: The hand to be deformed, Right: The deformed hand (with an added grid to show the deformation). Here, an affine regularisation has been used

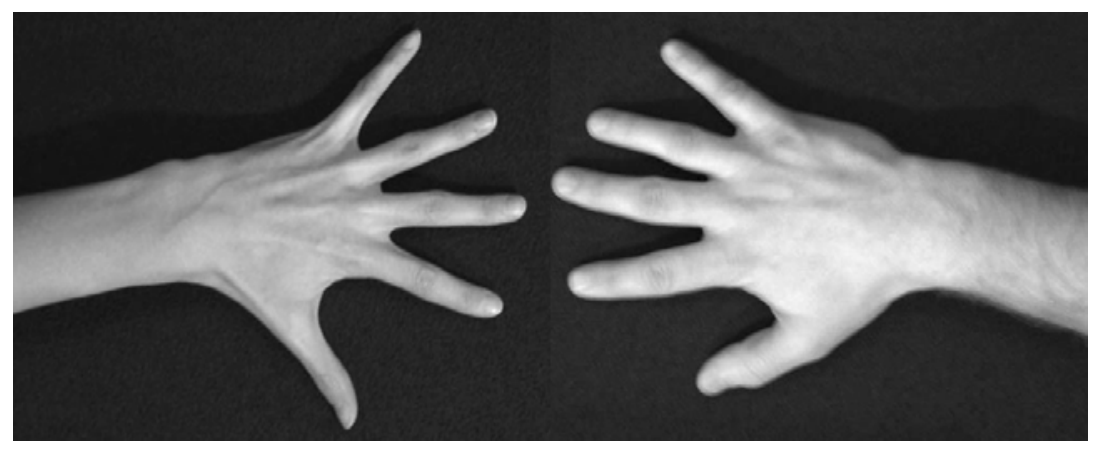

Fig. 4. The results from affine hand registration. Left: The registered hand (mirrored), Right: The 'real' hand

\section{Hips}

The hip example demonstrates the Morphon method applied to 3D CT data. The goal is to automatically segment the femoral bone and the pelvic bone as two separate objects from the data. Classification of tissue classes in CT images is usually done by simple thresholding, but due to osteoporosis, the bone density 

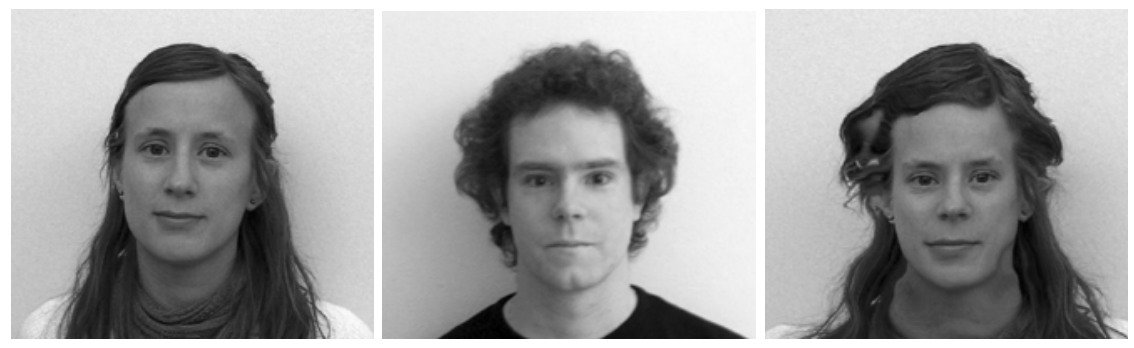

Fig. 5. Left: The image to deform, Middle: The target image, Right: The result after morphing the leftmost image onto the middle image
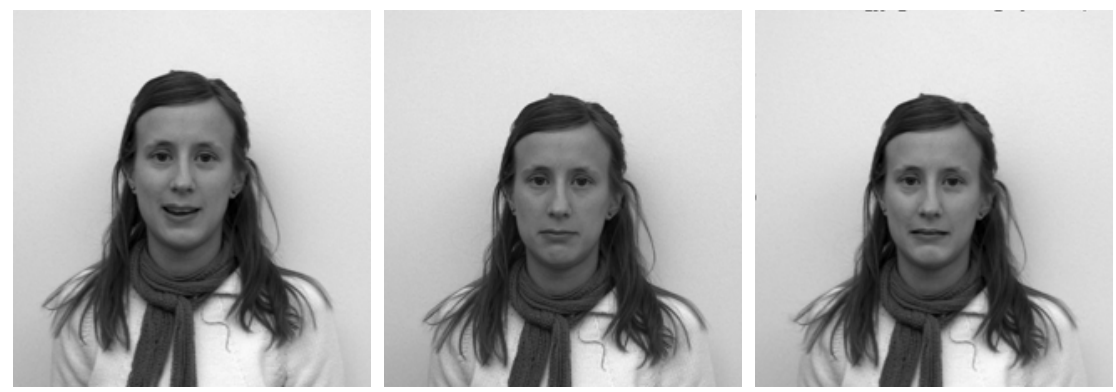

Fig. 6. Left: The prototype image with a happy expression, Middle: The target image with a sad expression, Right: Result from morphing the happy face onto the sad face
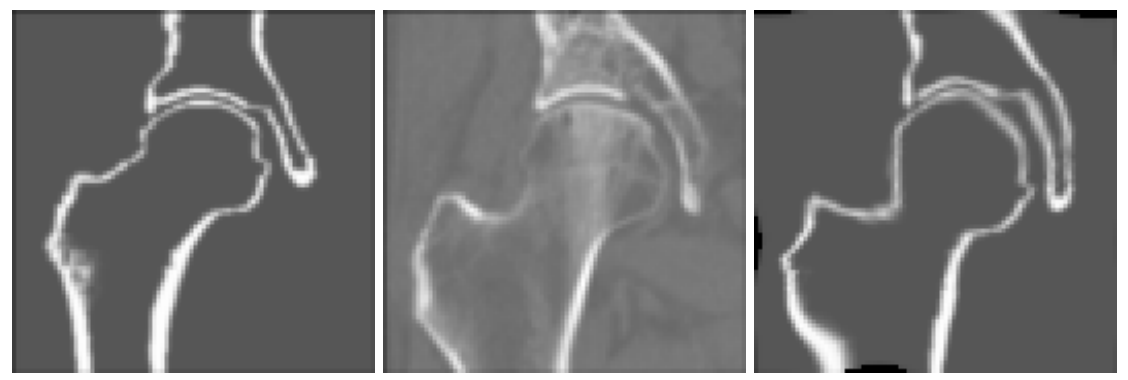

Fig. 7. 2D coronal slices of the hip data. Left: The hand-segmented data used as prototype, Middle: The CT data, Right: The prototype after deformation

is reduced to levels below that of the surrounding soft tissue. This gives CT images where the bone and the surrounding tissue have similar intensity levels, and where the border between the two is not always clearly distinguishable. Moreover, the joint space separating the pelvis and the femoral head is in most cases not possible to detect because of the low resolution of the image data. A prototype containing a hand segmented femur and pelvis, with basically two 


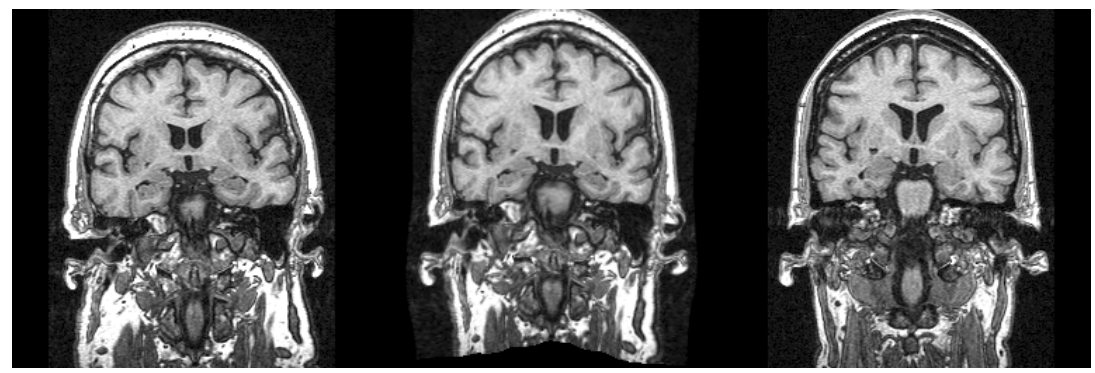

Fig. 8. Left: Prototype volume (brain a), Middle: Deformed prototype (brain a) Right: Target volume (brain b)

gray levels, is registered to the patient data. When the registration process is completed the femur and pelvis, now transformed into the shape of the specific patient, can be segmented from the deformed prototype. 2D coronal slices of the original prototype, the target data and the deformed prototype are shown in figure 7. A local transformation model has been used.

\section{Brains}

The final experiment was performed on 3D MRI-data of two brains. This is quite a challenge since no two brains look the same. One should most likely think carefully what one actually wishes to accomplish with such a registration. In this case we are interested only in the region around the left and right hippocampus. That is, we do not even wish to deform every winding section of the brains to look the same. This drastically reduces the complexity of the (still quite tricky) operation.

Figure 8 shows one section of the two original MRI volumes ( $a$ and $b$ ) along with the deformed version of the prototype volume (b).

\section{Discussion}

The application range for non-rigid registration techniques is wide, which explains the large amount of research devoted to this area. Deformation models, displacement estimation and similarity measures, are some relevant concepts associated to these methods. These concepts can be thought of as research areas of their own, and by combining these elements in new ways novel registration schemes can be found. The Morphon algorithm presented here can be characterized as a general registration method. It can be adapted to quite varying applications, which has been proven by the examples shown in this paper.

This paper does not, however, contain a comparison of the Morphon method to other well-known registration techniques, which is necessary to evaluate its functionality. In spite of this, we will point out some features that in much suggest that the Morphon method is a competitive registration algorithm. 
One advantage with the Morphon method is that it uses techniques which makes it robust in several senses. By using the quadrature phase as an input for measuring the similarity between the images, and obtaining the displacement estimation from the differences in the filter outputs, the algorithm becomes less sensitive to variations in the intensity of the data. This is beneficial in cases where the intensity level in the data is not closely related to the content of the data. One example of this is when working with photos, such as the hands or faces in the above examples. Due to different lighting conditions when obtaining the images, and difference in skin colour and reflectance, it would be very difficult to register these images to each other by only taking the gray values into account. However, in cases where the image intensity actually has a significance, such as in CT data where the intensity values directly correspond to the type of tissue, it would be disadvantageous not to utilize this knowledge. Thus, a method considering both the phase and the image intensity would be optimal in such cases.

Furthermore, because of the certainty measures included in the registration process the method becomes more stable. By applying these certainties as weights both in the estimation and the accumulation of the displacement fields the resulting deformation mainly depends on the neighbourhoods where the similarity between the images is large.

\section{Future Issues and Applications}

The general Morphon framework is definitely not limited to the implementations presented here. Since the Morphon components are fairly independent of each other, one can easily plug in new displacement estimation or regularisation methods when needed. Among the features one could imagine to incorporate is more prior information on the images. If e.g. images of hands are to be registered, a hand-specific deformation model would be reasonable.

The Morphon framework is here presented as a registration scheme. Its most likely application area would be to use such registration to perform atlas-based segmentation. All the prior information on the studied objects is incorporated in the prototype and morphed along with it. After registration to a target image, the morphed prior information can be used to draw conclusions about the target image.

As mentioned earlier, the Morphon in its current shape makes use of local measures to find a deformation field corresponding to a low cost. Most registration methods are based on a global energy measure of some kind which one would then attempt to minimise. Not seldom will the minimisation process involve a local formulation of some kind, meaning that the practical difference will not be enormous. It could, however, be beneficial to incorporate some kind of global measure also in the case of the Morphon. One problem now is that we do not consider whether the displacement estimates are made between structures that correspond to each other or to some completely different structure. To some extent this could be dealt with by the deformation model. In other cases we might wish to adapt the displacement estimation model itself to look at e.g. the likelihood of a certain voxel belonging to a certain tissue class or similar. 
Creating atlases is one very likely application for the Morphon method. By performing a number of user-guided Morphon registrations on a set of data, a database of typical deformation fields is obtained. This database can be used to create problem-specific prototypes where not only the intensity of the depicted objects is known, but also statistical models of shape variations similar to those used in active shape modeling.

\section{Conclusions}

The Morphon method was presented as a 2D and 3D non-rigid registration method. Its capabilities were successfully demonstrated on four very different applications. The results are promising but further evaluation, and comparison to existing registration techniques, must be done to assess its functionality. Finally a number of future extensions to adapt the Morphon method to very challenging problems such as atlas based segmentation were suggested.

\section{Acknowledgement}

We would like to thank Mats Andersson for interesting discussions on Morphons and more. We are also grateful to our project funders; Vetenskapsrådet, Vinnova and SSF, along with our research partners; Helge Malmgren at Göteborgs University and Melerit AB, Linköping.

\section{References}

1. J. L. Barron, D. J. Fleet, and S. S. Beauchemin. Performance of optical flow techniques. Int. J. of Computer Vision, 12(1):43-77, 1994.

2. G. Farnebäck. Polynomial Expansion for Orientation and Motion Estimation. PhD thesis, Linköping University, Sweden, SE-581 83 Linköping, Sweden, 2002. Dissertation No 790, ISBN 91-7373-475-6.

3. M. Felsberg. Disparity from monogenic phase. In L. v. Gool, editor, 24. DAGM Symposium Mustererkennung, Zürich, volume 2449 of Lecture Notes in Computer Science, pages 248-256. Springer, Heidelberg, 2002.

4. D. J. Fleet and A. D. Jepson. Computation of Component Image Velocity from Local Phase Information. Int. Journal of Computer Vision, 5(1):77-104, 1990.

5. G. H. Granlund and H. Knutsson. Signal Processing for Computer Vision. Kluwer Academic Publishers, 1995. ISBN 0-7923-9530-1.

6. M. Hemmendorff. Motion Estimation and Compensation in Medical Imaging. PhD thesis, Linköping University, Sweden, SE-581 85 Linköping, Sweden, 2001. Dissertation No 703, ISBN 91-7373-060-2.

7. B. K. P. Horn and B. G. Schunck. Determining optical flow. Artificial Intelligence, $17: 185-204,1981$.

8. J. P. W. Pluim, J. B. A. Maintz, and M. A. Viergever. Mutual-information-based registration of medical images: A survey. IEEE Transactions on Medical Imaging, 22(8):986-1004, 2003.

9. B. Zitova and J. Flusser. Image registration methods: a survey. Image and Vision Computing, 21(11):977-1000, October 2003. 